
\title{
Reseacch Soutere \\ Knockdown of miR-423-5p simultaneously upgrades the eNOS and VEGFa pathways in ADSCs and improves erectile function in diabetic rats
}

\section{JUN ZHOU}

Central South University Third Xiangya Hospital https://orcid.org/0000-0001-8695-1672

\section{Yinghao Yin}

Central South University Third Xiangya Hospital

\section{Yuan Yang}

Central South University Third Xiangya Hospital

\section{Dongyi Peng}

Central South University Third Xiangya Hospital

\section{Jingchao Wei}

Central South University Third Xiangya Hospital

Yuxin Tang ( $\sim$ mmcct@126.com )

\section{Research}

Keywords: Diabetes mellitus, erectile dysfunction, ADSCs, miR-423-5p, eNOS, VEGFa

Posted Date: February 4th, 2021

DOI: https://doi.org/10.21203/rs.3.rs-115770/v2

License: (c) (i) This work is licensed under a Creative Commons Attribution 4.0 International License. Read Full License 


\section{Abstract}

Background Erectile dysfunction (ED) is both physical and psychological problem to men. The treatment is still unsatisfied. Modified adipose derived stem cells (ADSCs) may be a good therapy. This study aim to investigate if knockout of miR-423-5p in ADSCs alleviates damage to endothelial cells in high glucose conditions and improves erectile function in diabetic rats.

Methods MiR-423-5p was knocked down in ADSCs. Then, ADSCs, NC-miR-ADSCs, and miR-ADSCs were co-cultured with human umbilical vein endothelial cells (HUVECs). Normal glucose and high glucose media were supplemented. Afterwards, the supernatant and HUVECs were collected for assessment of eNOS and VEGFa expression, cell proliferation, and apoptosis. Fifteen male Sprague-Dawley (SD) rats aged 12 weeks were induced to develop diabetes mellitus by intraperitoneal injection with streptozocin (STZ), and five healthy SD rats were used as normal controls. Eight weeks after developing diabetes, the rats received ADSCs and miR-ADSCs via injection into the corpora cavernosa, whereas normal controls and DM controls were injected with saline. Erectile function and histological assessment of penile tissues were performed eight weeks after injection.

Results MiR-423-5p was successfully inhibited in ADSCs, and HUVECs co-cultured with ADSCs or miRADSCs exhibited higher eNOS and VEGFa protein expression levels compared to DM groups $(P<0.05)$. MiR-ADSCs enhanced HUVEC proliferation compared to the ADSCs and NC-miR-ADSCs in both normal and high glucose conditions $(P<0.01)$. Lower apoptotic rates were observed when HUVECs were cocultured with miR-ADSCs, compared to ADSCs and NC-miR-ADSCs $(P<0.05)$. The ICP/MAP indicated that erectile function was severely impaired in the DM rats compared with the normal group. Injection of ADSCs and miR-ADSCs improved erectile function $(P<0.01)$ significantly and was associated with the overexpression of eNOS and VEGFa. Masson trichrome stain showed less fibrosis formation in the miRADSC group than the DM group.

Conclusions MiR-423-5p knockdown in ADSCs ameliorated high glucose-mediated damage to HUVECs and improved erectile function in DM rats by inducing eNOS and VEGFa overexpression, indicating that miR-423-5p may be a potential target in the treatment of DM erectile dysfunction (DMED).

\section{Background}

The morbidity and mortality of diabetes mellitus (DM), which is associated with chronic complications, are continuously increasing ${ }^{1,2}$. Erectile dysfunction (ED) is more likely to happen in men with $\mathrm{DM}^{3}$. In different populations and ages, the morbidity rate of ED among men with DM varies from $35 \%$ to $90 \% 4,5$. Diabetes mellitus-induced erectile dysfunction (DMED), which has pathogenic features that include endothelial, neuropathic and microvascular damage and fibrous-muscular alterations, is usually more severe and difficult to treat than nondiabetics, in disregard of the heavy burden ${ }^{6,7}$. 
Recently, several experimental approaches for DMED have been developed, including insulin treatment ${ }^{8}$, antioxidant therapy ${ }^{9}$, low energy shockwave therapy ${ }^{10}$, stem cells, and gene therapy ${ }^{11,12}$. Among these strategies, stem cell-based therapy is considered promising due to its ability to recover functional cells and tissues. In choosing the candidate stem cells from many kinds of mesenchymal stem cells, adiposederived stem cells (ADSCs) appear to be one of the most suitable types ${ }^{3,13}$. ADSCs have several biological benefits, including a large amount of autologous sources, ease in isolation, and the ability to expand ${ }^{14}$. The results of studies in rats with diabetes and cavernous injury, which were treated with the intracavernous injection of ADSCs, showed that erectile function had been restored. ${ }^{15-17}$.

Endothelial nitric oxide synthase (eNOS) plays a key role in penile erection. The blood flow-induced phosphatidylinositol 3-kinase/Akt/eNOS phosphorylation cascade reducing the calcium dependence and sustaining endothelial nitric oxide (NO) release and making the cavernous smooth muscle relax continuously, helps the penile erection maintenance. ${ }^{18}$. Endothelial and smooth muscle cells can secrete vascular endothelial growth factor (VEGF) which is a multifunctional glycoprotein. Receptor-mediated endothelial proliferation can be induced by VEGF in vitro and in vivo. And VEGF is related to endothelial

function and an effective vasculogenic and vascular permeability factor. ${ }^{19}$. Recently, studies have shown that endogenous NO production can be stimulated by VEGF, which can play positive roles on endothelial and smooth muscle cells, resulting in improvement in erectile function ${ }^{20,21}$.

MicroRNAs (miRs) are short non-coding RNA that are 18-23 nucleotides in length ${ }^{22}$. miRs function in regulating gene expression by binding to the $3^{\prime}$ untranslated region of the target mRNA ${ }^{23}$. In this study, bioinformatics analysis indicated that miR-423-5p has the ability to regulate both eNOS and VEGFa genes. Thus, we investigated whether miR-423-5p can improve endothelial cell function and ameliorate erectile function in DM rats, as both eNOS and VEGFa genes play key functions in the mechanism of penis erection. To our knowledge, this is the first study to investigate miRs regulating two important genes in a DMED study.

\section{Materials And Methods}

\section{Cell culture}

Adipose-derived stem cells (ADSCs) and HUVECs were purchased from Procell Life (Wuhan, China). ADSCs were maintained in stem cell culture medium supplemented with $10 \%$ fetal bovine serum and antibiotics (100 units/mL penicillin and $100 \mathrm{mg} / \mathrm{mL}$ streptomycin). HUVECs were maintained in endothelial cell basal medium supplemented with $1 \%$ endothelial cell growth supplement and $10 \%$ fetal bovine serum. The glucose concentration of high glucose medium was $30 \mathrm{mmol} / \mathrm{L}$. The ADSCs were transfected with CRISPR lentivirus-carrying miR-423-5p or miR-negative control (miR-NC) (Shanghai GenePharma Co., Ltd, Shanghai, China). ADSCs, miR-NC-ADSCs, and miR-ADSCs were co-cultured with HUVECs, and the supernatant was collected for enzyme-linked immunosorbent assay (ELISA).

\section{Cell apoptosis analysis}


Apoptosis was assessed with an Annexin V-FITC/PI Apoptosis Detection Kit (Beyotime Institute of Biotechnology, Shanghai, China) according to the manufacturer's instructions. Briefly, after treatment and incubation for $48 \mathrm{~h}$, the cells were collected, washed with PBS, and stained with Annexin $\mathrm{V}$ and propidium iodide (Ptdlns) in the dark using an Annexin V-FITC apoptosis detection kit (Beyotime Biotechnology, Shanghai, China). Cell apoptosis was subsequently analyzed by FACSCalibur flow cytometry (BD Biosciences, San Jose, CA, USA).

\section{Quantitative real-time PCR}

Total RNA was extracted from tissue samples or cells using TRIzol following the manufacturer's instructions. Total RNA was reverse transcribed into CDNA using a PrimeScript RT reagent kit. Reverse transcription polymerase chain reaction (RT-PCR) was performed using the iQ5 Multicolor Real-Time PCR Detection System (Bio-Rad Laboratories, Inc., Hercules, CA, USA) with SYBR Premix Ex Taq II. The PCR primer sequences for miR-423-5p and U6snRNA were as follows: miR-423-5p forward primer 5 'ACACTCCAGCTGGGTGAGGGGCAGAGAGCGA-3', reverse primer 5'-

CTCAACTGGTGTCGTGGAGTCGGCAATTCAGTTGAGAAAGTCTC-3'; U6snRNA forward primer 5'CTCGCTTCGGCAGCACA-3', and reverse primer 5'-AACGCTTCACGAATTTGCGT-3'. A melting curve analysis of the amplified products was performed at the end of each PCR cycle. U6snRNA was used as internal control, and gene expression was relatively quantified using the 2- $\Delta \Delta \mathrm{CT}$ method.

\section{Western blotting (WB)}

WB was performed as previously described. Briefly, the cells and rat penises were lysed in lysis buffer containing protease inhibitors. Protein concentrations of the lysates were determined by the bicinchoninic acid assay (Beyotime Biotechnology). Equal amounts of protein $(20 \mu \mathrm{g})$ were separated by $10 \%$ SDSPAGE and subsequently transferred onto PVDF membranes. The membranes were blocked with $5 \%$ nonfat dry milk in $0.2 \%$ Tween-20 in Tris-buffered saline (TBS-T) for $1 \mathrm{~h}$ at room temperature and then hybridized with primary antibodies. The primary antibodies were mouse anti-eNOS (1:400), mouse antiVEGFa (1:400), and mouse anti-glyceraldehyde phosphate dehydrogenase (GAPDH, 1:10,000). Immunoreactivity was detected after incubation with a horseradish peroxidase-conjugated secondary antibody according to the manufacturer's instructions (Thermo Scientific, Waltham, MA, USA). GAPDH was used as loading control. The positive bands were analyzed using Gel-pro analyzer software, and integrated optical density (IOD) was measured.

\section{ELISA}

The cell culture medium was collected after coculture, the supernatant was collected by centrifugation for 10 min at 1,500 rpm. eNOS and VEGFa expression levels were measured using an ELISA kit (Solarbio Life Sciences, Beijing, China) according to the manufacturer's instructions (SEA868Ra for eNOS, SEA143Ra for VEGFa). Absorption at a wavelength of $450 \mathrm{~nm}$ (A450) was determined using the microplate reader.

\section{Masson trichrome stain}


Masson trichrome staining using a Trichrome Stain (Masson) Kit (Sigma-Aldrich Co., St Louis, MO, USA) was performed to visualize fibers in tissues, following the manufacturer's instructions. Briefly, the tissue slides were deparaffinized, stained in preheated Bouin's solution, and washed in running tap water to remove the yellow color from sections. Then, the slides were respectively stained in Working Weigert's Iron Hematoxylin Solution, Biebrich Scarlet-Acid Fucshin, Working Phosphotungstic/Phosphomolybdic Acid Solution, and Aniline Blue Solution. The stained slides were observed under an optical microscope (magnification 40').

\section{Immunofluorescene staining}

For immunofluorescene staining (IF), the primary antibodies were mouse anti-eNOS (1:400), mouse antiVEGFa (1:400), secondary antibodies included Alexa-488-conjugated antibodies and Alexa-592conjugated antibodies (1:500), Nuclear staining was accomplished with 4',6-diamidino-2-phenylindole (DAPI).

\section{Intracavernosal pressure (ICP) measurement}

Erectile function was determined by intracavernosal pressure (ICP) and mean arterial pressure (MAP) 4 weeks post-injection. Under $3 \%$ pentobarbital sodium, the major pelvic ganglion (MPG) and cavernous nerves $(\mathrm{CN})$ were exposed by midline laparotomy. The penile was exposed by removing overlying skin and ischiocavernosus muscle. One of the 24-gauge needles that were connected to PE-50 tubes with heparinized saline (250 $\left.\mathrm{IU} \mathrm{ml}^{-1}\right)$ was inserted into the left carotid to measure MAP. The other one was inserted into corpus cavernosum (CC) to measure ICP. PE-50 tubes were connected to the data acquisition system (MP150, BIOPAC Systems Inc., Goleta, CA, USA). The CNs were stimulated using a stainless steel bipolar hook electrode with the following parameters: $20 \mathrm{~Hz}$, pulse width of $0.2 \mathrm{~ms}, 1.5 \mathrm{~mA}$, for $50 \mathrm{~s}$. The ratio of maximal ICP $(\mathrm{mm} \mathrm{Hg})$ to MAP $(\mathrm{mm} \mathrm{Hg})$ was calculated.

\section{Luciferase reporter assay}

PmirGLO-NOS3.3UTR and pmirGLO-VEGFA.3UTR were constructed. The miR-423-5p mimics and negative control sequence were as follows: 5'-UGAGGGGCAGAGAGCGAGACUUU-3' and 5'UUCUCCGAACGUGUCACGUTT-3'. pRL-TK vector (Takara Biotechnology Ltd., Dalian, China) of Renilla luciferase was used as internal reference for adjusting the differences in cell number and transfection efficiency. Approximately $2 \times 10^{4}$ cells were seeded into a 48-well plate individually and co-transfected with $500 \mathrm{ng}$ pmirGLO-NOS3.3UTR and pmirGLO-VEGFA.3UTR. Subsequently, the cells were transfected with mimics negative control and miR-423-5p mimics, respectively. After $48 \mathrm{~h}$ of transfection, the luciferase assay was conducted using a dual luciferase reporter assay (Promega, USA) according to the manufacturer's instructions.

\section{Statistical analysis}


All statistical analyses were performed with SPSS 19.0 (SPSS Inc., Chicago, IL, USA). All results are expressed as the mean \pm standard deviation (SD). Multiple comparisons between groups were performed using ANOVA followed by post hoc analysis using the Tukey-Kramer test, whereas a comparison between two groups was performed using a t-test. Differences with a $\mathrm{P}<0.05$ were considered statistically significant.

\section{Results}

\section{MiR-423-5p directly targets eNOS and VEGFa mRNAs}

Bioinformatics prediction revealed that miR-423-5p has one specific potential binding site for eNOS and VEGFa mRNAs within the 3'-UTR (Fig 1 A). There are seven base-pairs at the binding site for eNOS mRNA and miR-423-5p, and eight for VEGFa mRNA and miR-423-5P. A luciferase assay was performed to validate this prediction. Both eNOS and VEGFa luciferase activity were suppressed relative to the control; the suppression of eNOS was $66 \%$, whereas VEGFa was $60 \%$ (Fig 1 B). These findings indicate that eNOS and VEGFa are target genes of miR-423-5p.

\section{miR-423-5p knockout improves eNOS and VEGFa protein expression in ADSCs}

To confirm whether miR-423-5p can influence eNOS and VEGFa protein expression, miR-423-5p was knocked down in ADSCs. miR-423-5p expression decreased after transfection with the knockout virus. At 12 and $24 \mathrm{~h}$, miR-423-5p expression was markedly reduced (Fig $2 \mathrm{~A})(\mathrm{P}<0.001)$. To demonstrate the silencing effects of miR-423-5p on eNOS and VEGFa, total proteins in ADSCs were extracted for western blot analysis. Compared with the control group, eNOS and VEGFa expression levels significantly increased (Figs $2 B-D)(P<0.05)$. These results suggest that miR-423-5p inhibits protein expression of eNOS and VEGFa.

\section{Knocking out miR-423-5p in ADSCs alleviates high glucose-induced damage in HUVECs}

We transfected ADSCs with the miR-423-5p inhibition lentivirus, and then miR-ADSCs were co-cultured with HUVECs. In normal glucose conditions, ADSCs, NC-miR-ADSCs, and miR-ADSCs promoted eNOS and VEGFa expression in HUVEC conditioned media (Figs 3 A and B). miR-ADSCs increased the protein expression of eNOS and VEGFa under high glucose conditions compared to ADSCs and NC-miR-ADSCs (Figs $3 \mathrm{~A}$ and $\mathrm{B}$ ). A CCK-8 assay was performed to assess cell proliferation. miR-ADSCs promoted HUVEC proliferation under both normal and high glucose conditions (Fig $3 \mathrm{C}$ ).

eNOS and VEGFa are key proteins in penis erection. Therefore, western blotting was performed to evaluate eNOS and VEGFa expression. miR-423-5p inhibition in ADSCs was associated with increased HUVEC eNOS and VEGFa expression. This increase was more profound under high glucose concentrations than under normal glucose concentrations (Fig $3 \mathrm{D}-\mathrm{F}$ ). To further investigate whether the inhibition of miR-423-5p in ADSCs would benefit HUVECs, cell flow cytometry was also conducted. 
Propidium iodide $(\mathrm{Pl})$ was used to assess cell apoptosis. The number of late apoptotic cells caused by high glucose damage sharply decreased when co-cultured with miR-ADSCs (Fig $3 \mathrm{G}$ ).

\section{Erectile function assessment}

Figures $4 \mathrm{~A}$ and $\mathrm{B}$ show that the DM group had lower ICP and ICP/MAP ratios than the ADSCs and miRADSCs groups $(P<0.05)$. Interestingly, the miR-ADSC group exhibited a significant increase in the ICP curve and ICP/MAP ratios compared to the ADSC group $(P<0.05)$. To assess eNOS and VEGFa expression, the penises from all rat groups were collected for western blotting analysis (Figs $4 \mathrm{C}, \mathrm{D}$ and $\mathrm{E}$ ) and immunofluorescene staining (Fig $5 \mathrm{~A}, \mathrm{~B}$ ), which showed that the DM group had lower eNOS and VEGFa protein expression than the ADSC or miR-ADSC groups $(P<0.05)$. The ratio of smooth muscle:collagen staining, as determined by Masson's trichrome staining in penile tissues, was significantly lower in the DM group compared to the ADSC and miR-ADSC groups (Fig 5). The ADSC and miR-ADSC groups had a similar ratio of smooth muscle:collagen staining (Fig 5).

\section{Discussion}

eNOS and VEGFa expression are both important for penile erection. Nitric oxide (NO) released from endothelium or nerve terminals stimulates the local production of cyclic guanosine monophosphate (cGMP), resulting in corporal smooth muscle relaxation, increased blood supply to the penis, and penile erection ${ }^{24}$. Macrovasculopathy, microvasculopathy, and endothelial dysfunction are major factors that regulate blood flow in the penis ${ }^{3}$. Microvascular disease and endothelial cell dysfunction play essential roles in DMED. In the present study, a reduction in the number of smooth muscles in the corpus cavernosum was observed in diabetic rats. Moreover, diabetic rats showed a decrease in eNOS and VEGFa protein expression, compared to rats without diabetes.

Endothelial nitric oxide (eNOS) plays an essential role in penile erection. The neuronal NOS can release NO which can initiate the penile erection in a rapid, short-term calcium-dependent way. While, the blood flow-induced phosphatidylinositol 3-kinase/Akt/eNOS phosphorylation cascade make the full penile erection and maintenance of erection. Sustained release of endothelial NO induces relaxation of local smooth muscle cells ${ }^{18}$. Endothelial cells produce NO that helps maintain vascular integrity and enhance vasculogenesis ${ }^{25}$. Previous studies have shown that eNOS-based gene therapy restores impaired angiogenesis in rats ${ }^{26,27}$ and that adenovirus-mediated eNOS gene transfer can promote reendothelialization of blood vessel in injured rabbits ${ }^{28}$.

VEGFa is the most widely studied member of the VEGF family. Reduced VEGFa signaling and impaired angiogenesis as well as collateral blood vessel formation occur in patients with diabetes mellitus ${ }^{29}$. Several groups have shown that humans and rats with ED have lower VEGFa expression than normal humans and rats, suggesting VEGFa could be a target for the treatment of ED. VEGFa also enhances the proliferation of cavernous smooth muscle cells and endothelial cells, further supporting erectile function ${ }^{30}$. VEGFa can be induced by a NO synthesis pathway that also facilitates angiogenesis ${ }^{31}$. Impaired 
vasculogenesis was reported in eNOS knockout mice $\left(\mathrm{eNOS}^{-/}\right)^{32}$. Diminished wound healing was also detected due to reduced VEGF-mediated migration ${ }^{33}$.

ADSCs are somatic stem cells with multipotency and little immunogenicity ${ }^{34}$. Moreover, ADSCs have been used to treat many diseases including the repair of muscular tissue ${ }^{35}$. Several groups have investigated the feasibility and advantage of using ADSCs for ED therapy in rat models ${ }^{36,37}$. Implantation of ADSCs has been shown to significantly improve erection function in diabetic ED rats ${ }^{12}$.

MicroRNAs are small, non-coding RNAs that modulate gene expression by binding to the $3^{\prime} U T R$ of the target gene ${ }^{38}$. MicroRNAs affect many biological processes, including cell apoptosis, proliferation, and metabolism ${ }^{39}$. A microRNA may not have a single target gene, instead influencing multiple genes simultaneously. We hereby describe an approach for improving eNOS and VEGFa expression for potential treatment of DMED using microRNAs. MiR-423-5p can promote gluconeogenesis and hyperglycemia ${ }^{40}$. A computational biology study showed miR-423-5p was high express in the obesity and type 2 diabetes adipose $^{41}$. Both hyperglycemia and obesity are bad effect on erectile function.

In the present study, we found that miR-423-5p simultaneously affected the expression of both eNOS and VEGFa. Knockout of miR-423-5p expression in ADSCs was associated with an increase in eNOS and VEGFa expression. ADSCs may differentiate into local smooth muscle cells or endothelial cells to restore organ function. ADSCs can also exert a local effect by secreting cytokines and growth factors ${ }^{17,36}$. In this study, ELISA and western blot results demonstrated that HUVEC co-cultured with miR-ADSCs showed overexpression of eNOS and VEGFa. As these two proteins play a key role in erection, we injected miRADSCs and ADSCs into diabetes-induced ED rats to evaluate their effect on erection. Both miR-ADSC and ADSC treated diabetic rats had improvement in erectile function. The miR-ADSC treated group showed greater improvement than the ADSC treated group. Together, these findings indicate that knocking out miR-423-5p relieves the inhibition of eNOS and VEGFa expression in ADSCs, thereby supporting penile erection.

Our results were similar to previous studies in that we also used stem cells in treating DM rats. However, the present study has limitations, and our findings require further validation. We did not assess fibrosis factors that may cause tunica albuginea diseases such as Peyronie's disease. In addition, the mechanism by which ADSCs affect HUVECs warrants further investigation, including whether ADSCs secrete exosomes.

\section{Conclusions}

Knocking down miR-423-5p in ADSCs ameliorated the high glucose damage in HUVECs and improved erectile function in DM rats. There was an associated overexpression of eNOS and VEGFa, suggesting that miR-423-5p may be potentially used as a target in cell therapy for DM associated erectile dysfunction. 


\section{Abbreviations}

eNOS: endothelial nitric oxide synthase

NO: nitric oxide

VEGFa: vascular endothelial growth factor a

ADSCs: adipose derived stem cells

HUVECs: human umbilical vein endothelial cells

STZ: streptozocin

DM: diabetes mellitus

ED: erectile dysfunction

DMED: diabetes mellitus erectile dysfunction

ICP: intracavernosal pressure

MAP: mean arterial pressure

MPG: major pelvic ganglion

$\mathrm{CN}$ : cavernous nerves

CC: corpus cavernosum

\section{Declarations}

\section{Availability of data and materials}

All datasets used during the current study are available from the corresponding author on reasonable request.

\section{Acknowledgements}

This work was supported by the National Natural Science Foundation of China (No. 81571432 and No. 82071636).

\section{Competing interests}

All authors declare no competing financial interests.

\section{Funding}


This work was supported by the National Natural Science Foundation of China (No. 81571432 and No. 82071636).

\section{Author information}

Jun Zhou ${ }^{1}$, Yinghao Yin ${ }^{1}$, Yuan Yang ${ }^{1}$, Dongyi Peng ${ }^{1}$, Jingchao Wei ${ }^{1}$, Yuxin Tang ${ }^{\star 1,2,3}$

\section{Authors' contribution}

$\mathrm{JZ}$ did the cell culture and rat experiment and was the major contributor in writing the manuscript; $\mathrm{YH}$ accomplished the immunofluorescene staining; $Y Y$ and JW analyzed the data and bioinformatics data; DP helped the rat experiment; YT designed the manuscript and got the funding. All authors read and approved the final manuscript.

\section{Affiliation}

1 Department of Urology,The Third Xiangya Hospital of Central South University, Changsha, China 2 Department of Urology, Fifth Affiliated Hospital of Sun Yat-sen University, Zhuhai, China 3 Guangdong Provincial Key Laboratory of Biomedical Imaging, The Fifth Affiliated Hospital, Sun Yat-sen University, Zhuhai, Guangdong Province, China.

\section{Corresponding author}

Correspondence to Yuxin Tang.

\section{Ethics declarations}

The study was approved by the IRB of Third Xiangya Hospital, Central South University.

\section{Consent for publication}

Not applicable.

\section{References}

1. Zhang P, Zhang X, Brown J, Vistisen D, Sicree R et al. Global healthcare expenditure on diabetes for 2010 and 2030. Diabetes Res Clin Pract 2010; 87: 293-301.

2. Stefek M. Natural flavonoids as potential multifunctional agents in prevention of diabetic cataract. Interdisciplinary toxicology 2011; 4: 69-77.

3. Zhou F, Hui Y, Xin H, Xu YD, Lei HE et al. Therapeutic effects of adipose-derived stem cells-based microtissues on erectile dysfunction in streptozotocin-induced diabetic rats. Asian journal of andrology 2017; 19: 91-7. 
4. Kamenov ZA. A comprehensive review of erectile dysfunction in men with diabetes. Experimental and clinical endocrinology \& diabetes : official journal, German Society of Endocrinology [and] German Diabetes Association 2015; 123: 141-58.

5. Malavige LS, Levy JC. Erectile dysfunction in diabetes mellitus. The journal of sexual medicine 2009; 6: $1232-47$.

6. Hatzimouratidis K, Hatzichristou D. How to treat erectile dysfunction in men with diabetes: from pathophysiology to treatment. Current diabetes reports 2014; 14: 545.

7. Thorve VS, Kshirsagar AD, Vyawahare NS, Joshi VS, Ingale KG et al. Diabetes-induced erectile dysfunction: epidemiology, pathophysiology and management. Journal of diabetes and its complications 2011; 25: 129-36.

8. Wang L, Tian W, Uwais Z, Li G, Li H et al. AGE-breaker ALT-711 plus insulin could restore erectile function in streptozocin-induced type 1 diabetic rats. The journal of sexual medicine 2014; 11: 145262.

9. Qiu XF, Li XX, Chen Y, Lin HC, Yu W et al. Mobilisation of endothelial progenitor cells: one of the possible mechanisms involved in the chronic administration of melatonin preventing erectile dysfunction in diabetic rats. Asian journal of andrology 2012; 14: 481-6.

10. Liu J, Zhou F, Li GY, Wang L, Li HX et al. Evaluation of the effect of different doses of low energy shock wave therapy on the erectile function of streptozotocin (STZ)-induced diabetic rats. International journal of molecular sciences 2013; 14: 10661-73.

11. Sun C, Lin H, Yu W, Li X, Chen Y et al. Neurotrophic effect of bone marrow mesenchymal stem cells for erectile dysfunction in diabetic rats. International journal of andrology 2012; 35: 601-7.

12. Qiu X, Sun C, Yu W, Lin H, Sun Z et al. Combined strategy of mesenchymal stem cell injection with vascular endothelial growth factor gene therapy for the treatment of diabetes-associated erectile dysfunction. Journal of andrology 2012; 33: 37-44.

13. Fandel TM, Albersen M, Lin G, Qiu X, Ning H et al. Recruitment of intracavernously injected adiposederived stem cells to the major pelvic ganglion improves erectile function in a rat model of cavernous nerve injury. European urology 2012; 61: 201-10.

14. Morizono K, De Ugarte DA, Zhu M, Zuk P, Elbarbary A et al. Multilineage cells from adipose tissue as gene delivery vehicles. Human gene therapy 2003; 14: 59-66.

15. Baraniak PR, McDevitt TC. Stem cell paracrine actions and tissue regeneration. Regenerative medicine 2010; 5: 121-43.

16. Hoke NN, Salloum FN, Kass DA, Das A, Kukreja RC. Preconditioning by phosphodiesterase-5 inhibition improves therapeutic efficacy of adipose-derived stem cells following myocardial infarction in mice. Stem cells 2012; 30: 326-35.

17. Garcia MM, Fandel TM, Lin G, Shindel AW, Banie L et al. Treatment of erectile dysfunction in the obese type 2 diabetic ZDF rat with adipose tissue-derived stem cells. The journal of sexual medicine 2010; 7: 89-98. 
18. Hurt KJ, Musicki B, Palese MA, Crone JK, Becker RE et al. Akt-dependent phosphorylation of endothelial nitric-oxide synthase mediates penile erection. Proceedings of the National Academy of Sciences of the United States of America 2002; 99: 4061-6.

19. Uccelli A, Wolff T, Valente P, Di Maggio N, Pellegrino M et al. Vascular endothelial growth factor biology for regenerative angiogenesis. Swiss medical weekly 2019; 149: w20011.

20. Ryu JK, Cho CH, Shin HY, Song SU, Oh SM et al. Combined angiopoietin-1 and vascular endothelial growth factor gene transfer restores cavernous angiogenesis and erectile function in a rat model of hypercholesterolemia. Molecular therapy : the journal of the American Society of Gene Therapy 2006; 13: 705-15.

21. Shirai M, Yamanaka M, Shiina H, Igawa M, Kawakami T et al. Vascular endothelial growth factor restores erectile function through modulation of the insulin-like growth factor system and sex hormone receptors in diabetic rat. Biochemical and biophysical research communications 2006; 341: 755-62.

22. Luppi F, Beghe B, Richeldi L. Acute exacerbations of chronic obstructive pulmonary disease: are antibiotics needed? American journal of respiratory and critical care medicine 2010; 181: 102-3.

23. Sun Y, Lu CM, Song Z, Xu KK, Wu SB et al. Expression and regulation of microRNA-29a and microRNA-29c in early diabetic rat cataract formation. International journal of ophthalmology 2016; 9: $1719-24$.

24. Mas M. Molecular mechanisms of penile erection. Archivos espanoles de urologia 2010; 63: 589-98.

25. Oladipupo SS, Smith C, Santeford A, Park C, Sene A et al. Endothelial cell FGF signaling is required for injury response but not for vascular homeostasis. Proceedings of the National Academy of Sciences of the United States of America 2014; 111: 13379-84.

26. Smith RS, Jr., Lin KF, Agata J, Chao L, Chao J. Human endothelial nitric oxide synthase gene delivery promotes angiogenesis in a rat model of hindlimb ischemia. Arteriosclerosis, thrombosis, and vascular biology 2002; 22: 1279-85.

27. Tai MH, Hsiao M, Chan JY, Lo WC, Wang FS et al. Gene delivery of endothelial nitric oxide synthase into nucleus tractus solitarii induces biphasic response in cardiovascular functions of hypertensive rats. American journal of hypertension 2004; 17: 63-70.

28. Sharif F, Hynes SO, Cooney R, Howard L, McMahon J et al. Gene-eluting stents: adenovirus-mediated delivery of eNOS to the blood vessel wall accelerates re-endothelialization and inhibits restenosis. Molecular therapy : the journal of the American Society of Gene Therapy 2008; 16: 1674-80.

29. Simons M. Angiogenesis, arteriogenesis, and diabetes: paradigm reassessed? Journal of the American College of Cardiology 2005; 46: 835-7.

30. Liu X, Lin CS, Graziottin T, Resplande J, Lue TF. Vascular endothelial growth factor promotes proliferation and migration of cavernous smooth muscle cells. The Journal of urology 2001; 166 : 354-60.

31. Sunshine SB, Dallabrida SM, Durand E, Ismail NS, Bazinet L et al. Endostatin lowers blood pressure via nitric oxide and prevents hypertension associated with VEGF inhibition. Proceedings of the 
National Academy of Sciences of the United States of America 2012; 109: 11306-11.

32. Dai X, Faber JE. Endothelial nitric oxide synthase deficiency causes collateral vessel rarefaction and impairs activation of a cell cycle gene network during arteriogenesis. Circulation research 2010; 106: 1870-81.

33. Aicher A, Heeschen C, Mildner-Rihm C, Urbich C, Ihling C et al. Essential role of endothelial nitric oxide synthase for mobilization of stem and progenitor cells. Nature medicine 2003; 9: 1370-6.

34. Bunnell BA, Flaat M, Gagliardi C, Patel B, Ripoll C. Adipose-derived stem cells: isolation, expansion and differentiation. Methods 2008; 45: 115-20.

35. Alexeev V, Arita M, Donahue A, Bonaldo P, Chu ML et al. Human adipose-derived stem cell transplantation as a potential therapy for collagen Vl-related congenital muscular dystrophy. Stem cell research \& therapy 2014; 5: 21.

36. Albersen M, Fandel TM, Lin G, Wang G, Banie L et al. Injections of adipose tissue-derived stem cells and stem cell lysate improve recovery of erectile function in a rat model of cavernous nerve injury. The journal of sexual medicine 2010; 7: 3331-40.

37. Yang J, Zhang Y, Zang G, Wang T, Yu Z et al. Adipose-derived stem cells improve erectile function partially through the secretion of IGF-1, bFGF, and VEGF in aged rats. Andrology 2018; 6: 498-509.

38. Huang K, Dong X, Sui C, Hu D, Xiong T et al. MiR-223 suppresses endometrial carcinoma cells proliferation by targeting IGF-1R. American journal of translational research 2014; 6: 841-9.

39. Shi Z, An N, Lu BM, Zhou N, Yang SL et al. Identification of novel kinase inhibitors by targeting a kinase-related apoptotic protein-protein interaction network in HeLa cells. Cell proliferation 2014; 47 : 219-30.

40. Yang W, Wang J, Chen Z, Chen J, Meng Y et al. NFE2 Induces miR-423-5p to Promote Gluconeogenesis and Hyperglycemia by Repressing the Hepatic FAM3A-ATP-Akt Pathway. Diabetes 2017; 66: 1819-32.

41. Gottmann P, Ouni M, Saussenthaler S, Roos J, Stirm L et al. A computational biology approach of a genome-wide screen connected miRNAs to obesity and type 2 diabetes. Molecular metabolism 2018; 11: $145-59$.

\section{Figures}


A

\begin{tabular}{|c|c|}
\hline & $\begin{array}{l}\text { Predicted consequential pairing of target } \\
\text { region (top) and miRNA (bottom) }\end{array}$ \\
\hline Position $220-226$ of NOS3 3'UTR & $5^{\prime} \quad \ldots$ GCCUUAAUCUGGAAGGCCCCUCC...3' \\
\hline & 1111111 \\
\hline hsa-miR-423-5p & UUUCAGAGCGAGAGACGGGGAGU 5 \\
\hline Position1447-1484 of VEGFa 3'UTR & .... CTAACACTCAGCTCTGCCCTCCCCG...3' \\
\hline & 11111111 \\
\hline hsa-miR-423-5p & UUUCAGAGCGAGAGACGGGGAGU 5 ' \\
\hline
\end{tabular}

B

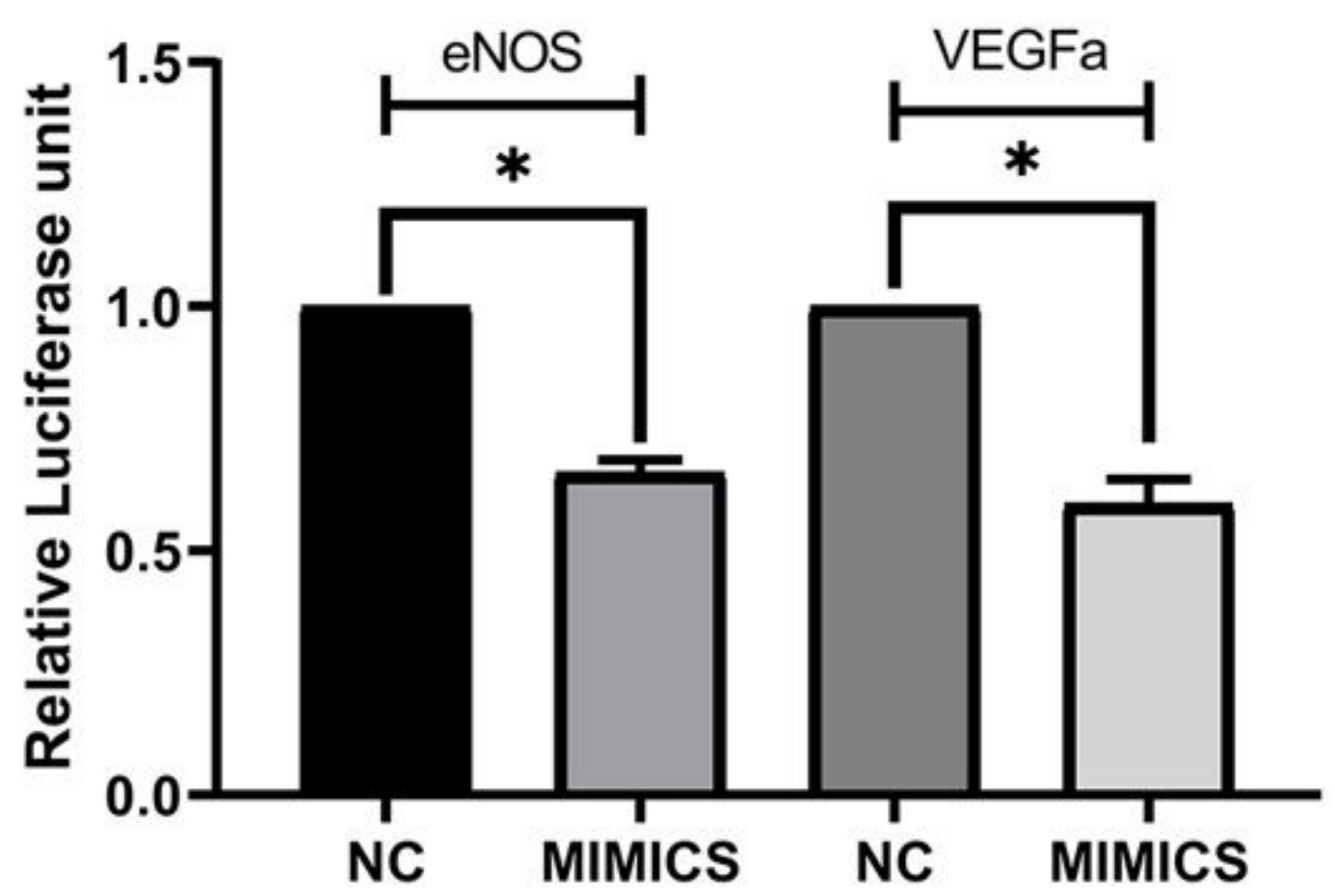

Figure 1

NOS3 and VEGFa are the target genes of miR-423-5p. A, Predicted miR-423-5p binding sites of the NOS3 and VEGFa genes are shown in red. B, Luciferase reporter assay showing post-transcriptional repression of the NOS3 and VEGFa genes. Cells were cotransfected with miR-423-5p mimics and pmirGLONOS3.3UTR or pmirGLO-VEGFA.3UTR cloned into expression vector downstream of the luciferase gene. 


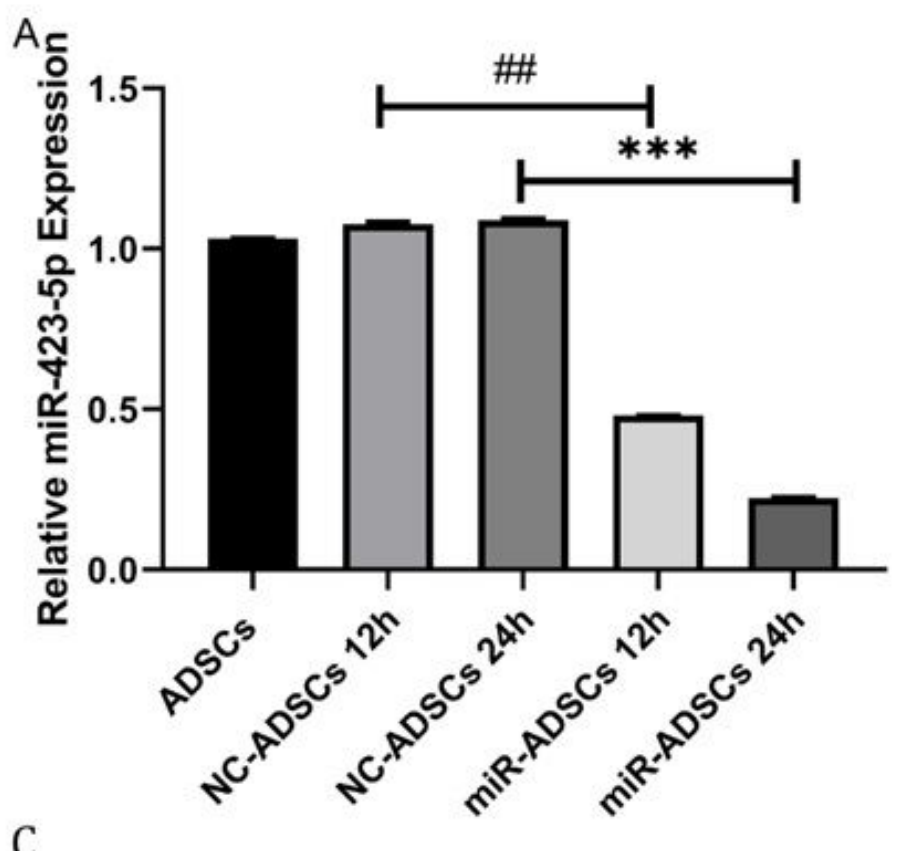

B

C
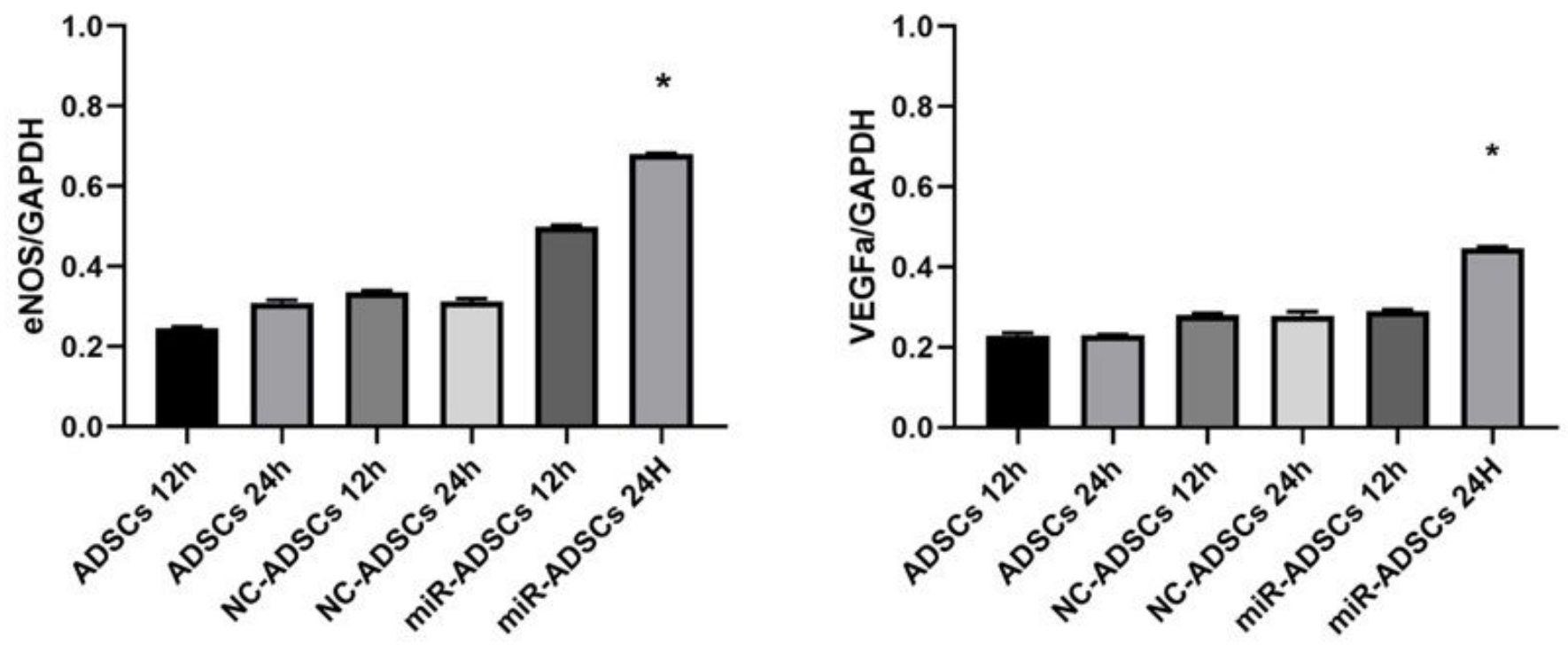

Figure 2

(A) Relative miR-423-5p expression after miR-423-5p knockdown in ADSCs. Approximately 12 and $24 \mathrm{~h}$ after inhibition of miR-423-5p, RNA was extracted for qRT-PCR analysis. miR-423-5p expression was downregulated at both 12 and $24 \mathrm{~h}(\mathrm{p}<0.001)$. (B) Western blot: eNOS and VEGFA expression after miR423-5p knockdown in ADSCs. (C and D) Relative eNOS and VEGFa expression levels increased with miR423-5p inhibition. $P<0.05$ vs. normal ADSCs. 

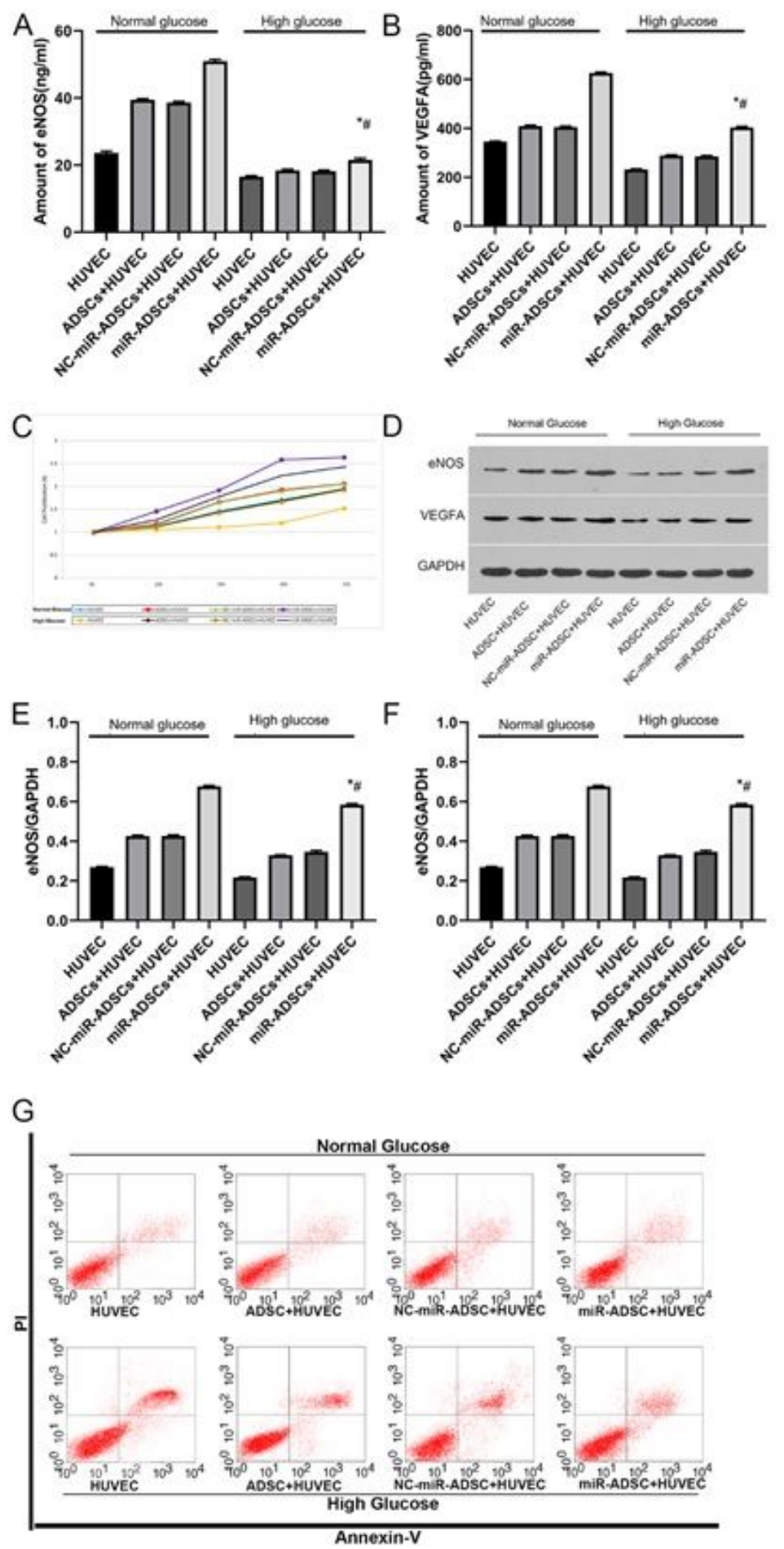

\section{Figure 3}

Changes in cell function when HUVECs are co-cultured with ADSCs, NC-miR-ADSCs, and miR-ADSCs in normal or high glucose supplemented media. (A) eNOS expression in the culture medium; (B) VEGFa expression in the culture medium; (C) cell proliferation; (D) eNOS and VEGFa protein expression levels; (E) relative eNOS protein expression levels; (F) relative VEGFa protein expression levels; (G) flow cytometry; y axis is propidium lodide( $\mathrm{PI}), \mathrm{x}$ is Annexin $\mathrm{V}$. The upper right quadrant represents late apoptotic and 
necrotic cells, while the lower right quadrant represents early apoptotic cells. * $p<0.01$ compared to miRADSCs+HUVEC in normal glucose conditions; \# $p<0.05$ compared to NC-miR-ADSCs+HUVEC in high glucose conditions.

A

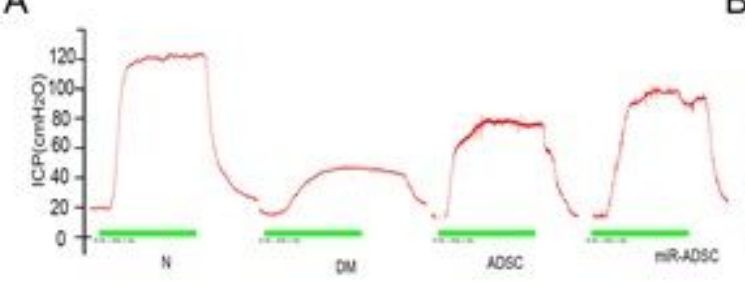

C

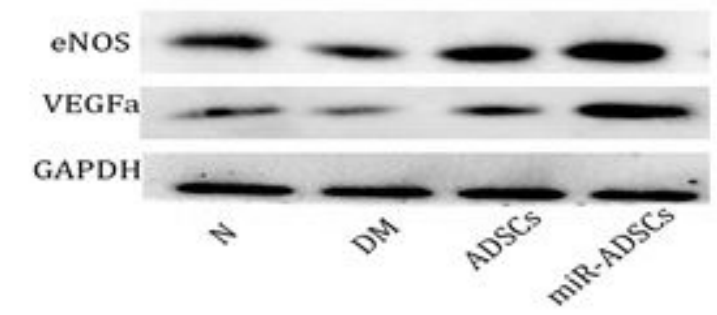

$\mathrm{E}$

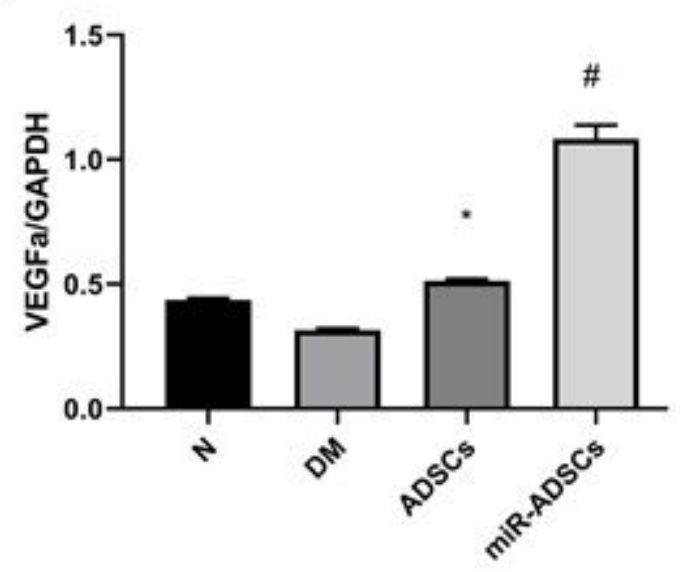

B
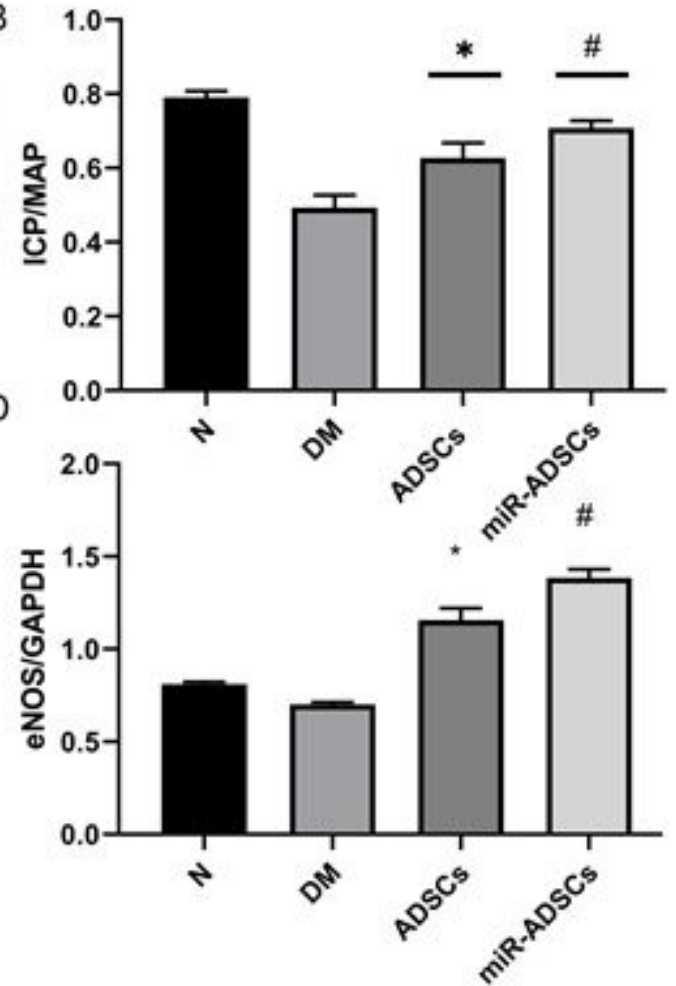

\section{Figure 4}

MiR-ADSCs improve erectile function upon cavernous nerve electrostimulation in diabetic SD rats. (A) Representative tracings of Intracavernosal Pressure (ICP) in $\mathrm{cm} \mathrm{H} 2 \mathrm{O}$ for normal rats $(\mathrm{N})$, diabetes mellitus rats (DM), ADSC-injected rats (ADSCs) and miR-ADSC-injected rats (miR-ADSC). (B) Erectile function is presented as the ICP/MAP ratio in each group. * $p<0.05$ compared to the DM group; \# $p<0.05$ compared to the DM group. (C) eNOS and VEGFa protein expression levels; (D) relative eNOS protein expression levels, * $p<0.05$ compared to the DM group, \# $p<0.05$ compared to the ADSC group; (E) relative VEGFa protein expression levels, ${ }^{*} p<0.05$ compared to the DM group, $\# p<0.05$ compared to the ADSC group; 
A
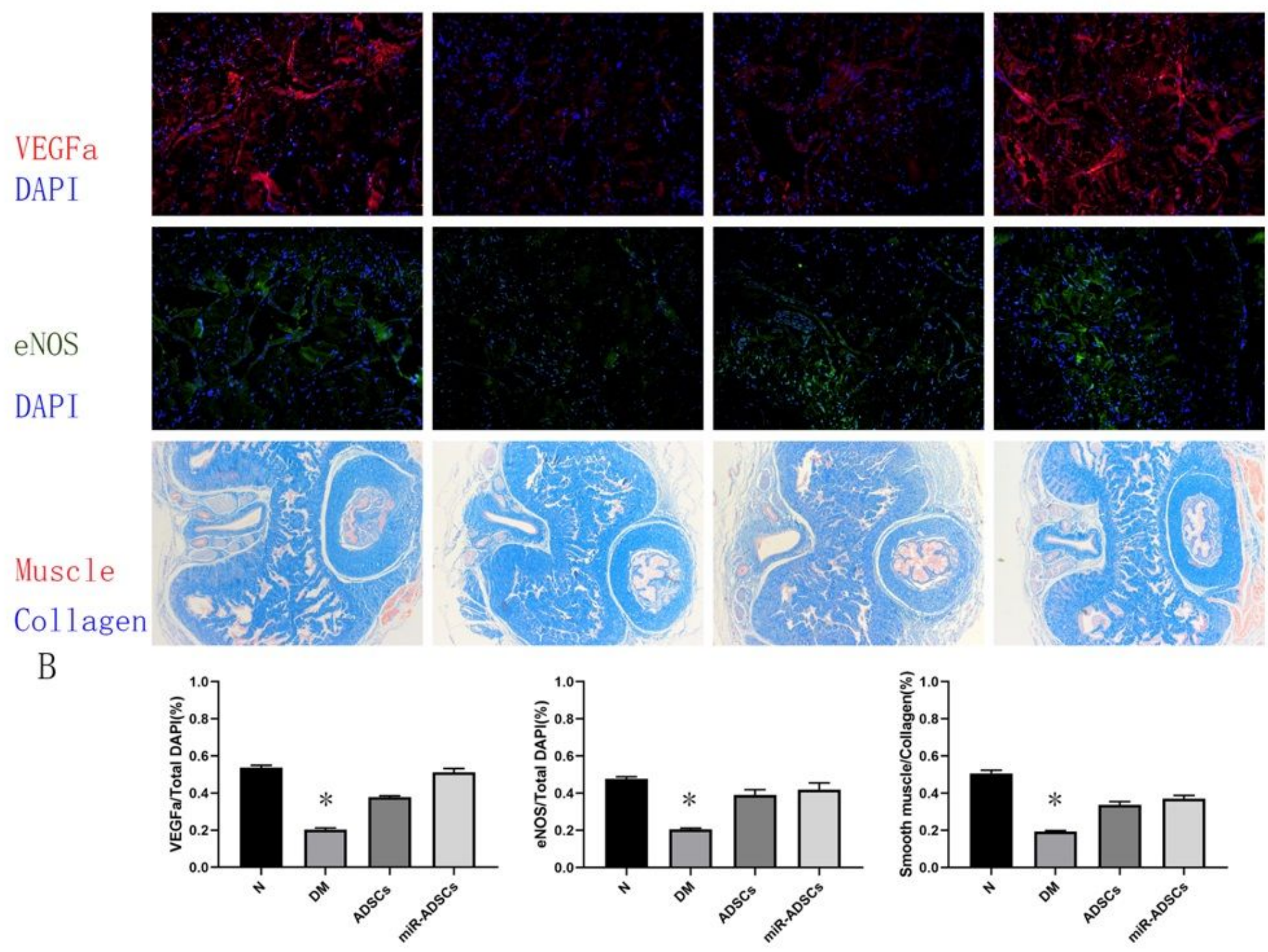

\section{Figure 5}

Expression of VEGFa and eNOS in the penile cavernous tissue 12 weeks after ADSCs/miR-ADSCs implantation. (A) Expression of VEGFa and eNOS assessed by immunofluorescene staining and the mason trichrome staining of the cavernous tissue. (B) Comparison of VEGFa and eNOS expression and the ratio of muscle and collagen in different treatment groups. ${ }^{*} p<0.05$ compared to the ADSCs group and miR-ADSCs group. 